\title{
Análise do consumo lipídico de idosos residentes da Região do Vale dos Sinos (RS)
}

\author{
Daiana da Silva*, Fernanda Ruppenthal", Flávia Porto Wieck", Denise Ruttke Dillenburg Osório***
}

\section{Resumo}

Estudo transversal e retrospectivo, com o objetivo de conhecer o consumo dietético de ácidos graxos em idosos, correlacionando com sexo, idade, cidade de residência e estado nutricional. Foram utilizados 526 prontuários de idosos participantes de uma pesquisa intitulada "Envelhecimento e Qualidade de Vida: Um Estudo em Municípios Selecionados do Vale dos Sinos, RS", os quais, após serem avaliados pelos critérios de inclusão e exclusão, resultaram em 482 prontuários utilizados neste estudo. A pesquisa foi realizada entre 2011 e 2014 com idosos de ambos os sexos, idade entre 60-79 anos, residentes de Campo Bom, Estância Velha ou Novo Hamburgo - RS. Foram avaliados dados sociodemográficos, antropométricos e consumo alimentar (R24h). A amostra caracterizou-se predominantemente pelo sexo feminino $(84,0 \%)$, idosos aposentados $(87,3 \%)$, renda de dois a cinco salários mínimos $(72,0 \%)$, escolaridade inferior a ensino fundamental completo $(70,5 \%)$, menos de cinco anos de estudo $(41,4 \%)$, moram acompanhados $(35,7 \%)$, casa própria $(93,2 \%)$, predominância de estado nu- tricional excesso de peso $(55,0 \%)$ e risco muito aumentado para doenças cardiovasculares $(81,5 \%)$. Podemos considerar após a análise dos resultados, que o consumo de ácidos graxos dos idosos da região estudada, estava adequado dentro da faixa de normalidade, com exceção para a relação de consumo de ômega 6:3. A cidade de Campo Bom, mostrou melhores níveis de consumo de ácido graxo em todas as frações lipídicas, com resultados significativos para a relação de consumo ômega 6:3 $(p=0,002)$. As demais variáveis não apresentaram resultados significativos em relação ao consumo lipídico.

Palavras-chave: Ácidos graxos. Doenças cardiovasculares. Idosos.

\section{Introdução}

Segundo uma projeção realizada pelo Instituto Brasileiro de Geografia e Estatística (IBGE), em agosto de 2013, a população idosa está crescendo de

* Bacharel em Nutrição na Universidade Feevale, Novo Hamburo, RS. Endereço para correspondência: Rua Alberto Dorneles, 25, Estação, Capela de Santana, RS. CEP: 95745-000. E-mail: daianad.s@hotmail.com

** Bacharel em Nutrição na Universidade Feevale, Novo Hamburgo, RS. E-mail: fernandaahruppenthal@ gmail.com

**** Bacharel em Nutrição e mestre em Gerontologia Biomédica (PUCRS). E-mail: portofla@gmail.com

**** Bacharel em Nutrição e mestre e doutora em Ciências da Saúde (Cardiologia) (IC/FUC-RS). Docente do Curso de Nutrição da Universidade Feevale, Novo Hamburgo, RS. E-mail: deniseosorio@feevale.br

$\rightarrow$ http://dx.doi.org/10.5335/rbceh.v14i3.7626

Recebido em: 13.11.2017. Aceito em: 03.04.2018. 
forma gradual e contínua. Dessa forma, enquanto a proporção de jovens diminui gradativamente, atingindo $13,0 \%$ da população brasileira, a expectativa de vida para o grupo de idosos (60 anos ou mais) passará de 13,8\%, em 2020, para $33,7 \%$, em 2060. Esses dados evidenciam claramente o processo de envelhecimento populacional do país (IBGE, 2013). Com esse panorama, haverá uma maior proporção de pessoas com aumento na expectativa de vida e, assim, uma maior probabilidade dessas desenvolverem doenças crônicas não transmissíveis (BEZERRA; ALMEIDA; THERRIEN-NÓBREGA 2012).

$\mathrm{O}$ processo de envelhecimento tem grande impacto na saúde e na qualidade de vida do idoso, dependente, em grande parte, de uma alimentação adequada e equilibrada. Quando não há esse equilíbrio, aumentam as chances do surgimento de doenças crônicas não transmissíveis, incluindo as alterações cardiovasculares. Relacionado a todos esses eventos, dá-se atenção principalmente para o consumo de lipídeos saturados e insaturados, além de colesterol, pois quando consumidos de maneira inadequada, são um grande fator de risco para o desenvolvimento dos eventos citados (KUCERA; SIVIERO; BONATTO, 2012).

Atualmente, as Doenças Cardiovasculares (DCV) são a principal causa de morte no Brasil. De acordo com dados da Pesquisa Nacional em Saúde, em 2013, $4,2 \%$ (6,1 milhões) da população brasileira com 18 anos ou mais, obtiveram diagnóstico para DCVs. Essa pesquisa mostrou ainda que o número de pessoas com doenças coronarianas aumenta de acordo com a idade, evidenciando-se uma probabilidade de desenvolvimento de doenças cardíacas duas vezes maior a cada década de vida, uma vez que são doenças que se iniciam na juventude, conforme hábitos de vida e manifestam suas consequências na vida adulta e idosa (NEVES et al., 2013).

Embora ainda sejam necessários mais estudos relacionando os eventos cardiovasculares com o padrão alimentar dos indivíduos, pode-se afirmar que a qualidade alimentar dos ácidos graxos ingeridos estão diretamente relacionados ao aumento do risco cardiovascular. O consumo inadequado de gorduras pode acelerar a formação de placas ateroscleróticas, aumentando os níveis de lipídeos plasmáticos, pressão arterial, entre outros eventos que contribuem para a formação de ateromas (SANTOS et al., 2013).

Os ácidos graxos são classificados de acordo com suas características químicas (saturados, poli-insaturados e monoinsaturados), sendo esse o fator que distingue o efeito que cada fração lipídica irá provocar no organismo, de modo que os ácidos graxos saturados irão provocar aumento no colesterol total e LDL-c, diminuindo HDL-c, e os poli-insaturados e monoinsaturados ocasionarão diminuição dos níveis plasmáticos de colesterol total e LDL-c, aumentando os níveis de HDL-c. Sendo assim, percebe-se a importância do consumo equilibrado de ácidos graxos, pois existe uma relação direta com o risco de eventos cardiovasculares, alterações 
nos níveis plasmáticos de lipoproteínas e colesterol total (SANTOS et al., 2013).

Diante do exposto, este estudo visa conhecer e verificar a prevalência de adequação do consumo dietético de ácidos graxos em idosos residentes nas cidades de Novo Hamburgo, Campo Bom e Estância Velha, localizadas na região do Vale dos Sinos, Rio Grande do Sul/ Brasil, correlacionando o consumo lipídico com sexo, idade, cidade de residência, estado nutricional e risco cardiovascular.

\section{Materiais e métodos}

Trata-se de um estudo transversal e retrospectivo. A amostra deste estudo fazia parte de um banco de dados, oriundos de uma pesquisa intitulada "Envelhecimento e qualidade de vida: um estudo em municípios selecionados do Vale dos Sinos, RS”. Todos os indivíduos desta pesquisa participavam de grupos de convivência da terceira idade e residiam em cidades próximas à universidade em que aconteceu a pesquisa. As cidades em que os sujeitos residiam eram, Campo Bom, Novo Hamburgo e Estância Velha, todas situadas na região do Vale dos Sinos - RS, resultando em uma amostra não probabilística, composta inicialmente por uma população de 526 prontuários de idosos de ambos os sexos. Os critérios de inclusão foram idade, acima de 60 anos, ser independentes para a realização das atividades diárias e residir em uma das cidades supracitadas. Excluíram-se deste estudo os questionários preenchidos de maneira incorreta ou que apresentaram informações imprecisas ou incompletas.

A coleta de dados ocorreu no período de agosto de 2011 a novembro de 2014 , em uma clínica escola situada na Universidade Feevale, Novo Hamburgo - RS. Todos os idosos que participaram do projeto eram isentos de qualquer custo e respondiam a um questionário sociodemográfico (idade, sexo, escolaridade, tempo de estudo, renda, se aposentados, local e tipo de residência, com quem reside), IMC e circunferência da cintura, com o intuito de identificar a amostra coletada quanto às características gerais e cidade em que residiam. Após, eram encaminhados para outras etapas, como, Avaliação Antropométrica e de Consumo Alimentar, a qual consistiu na aplicação de um Recordatório Alimentar de 24 horas (R24h). Para a verificação da antropometria, foi recomendado que os idosos permanecessem apenas com as roupas íntimas, jaleco fornecido pela clínica e descalços, para que estes fatores não comprometessem e interferissem no resultado final. $\mathrm{O}$ peso e a altura foram aferidos em balança antropométrica digital com estadiômetro acoplado, da marca Welmy ${ }^{\circledR}$, com capacidade máxima de $200 \mathrm{Kg}$. A circunferência da cintura foi determinada por meio de uma fita métrica, não extensível, a partir do ponto médio entre o rebordo costal inferior e a crista ilíaca. Essa medida foi utilizada para a classificação do risco de complicações metabólicas de acordo com o estado nutricional (ABESO, 2009-2010). Todos os procedimentos foram realizados por estudantes do curso de nutrição aptos e 
supervisionados por professora responsável. O estado nutricional da população estudada foi obtido por meio do parâmetro de Índice de Massa Corporal (IMC) para idosos, de acordo com Opas (2003) e para classificação do risco de doenças cardiovasculares (DCV) foi utilizada circunferência da cintura, seguindo os pontos de corte encontrados em Herdy et al. (2014), conforme Quadro 1.

Quadro 1 - Pontos de corte utilizados para classificação do IMC e circunferência da cintura

\begin{tabular}{|l|l|l|l|}
\hline \multicolumn{1}{|c|}{ IMC } & \multicolumn{1}{|c|}{ Circunferência da cintura } & \multicolumn{1}{c|}{ Pontos de corte } \\
\hline Classificação & Pontos de corte & \multicolumn{1}{c|}{ Classificação } & $\begin{array}{l}<80 \mathrm{~cm} \text { para mulheres } \\
<94 \mathrm{~cm} \text { para homens }\end{array}$ \\
\hline Baixo peso & $\leq 23$ & Adequado & $\begin{array}{l}\geq 80-<88 \text { para mulheres } \\
\geq 94-<102 \text { para homens }\end{array}$ \\
\hline Eutrofia & $>23-<28$ & Risco aumentado para DVC & $\begin{array}{l}\geq 88 \text { para mulheres } \\
\geq 102 \text { para homens }\end{array}$ \\
\hline Sobrepeso & $\geq 28-<30$ & Risco muito aumentado para DVC & \\
\hline Obesidade & $\geq 30$ & & \\
\hline
\end{tabular}

Fonte: elaboração dos autores.

Para avaliar o consumo dos ácidos graxos, foi utilizado software de nutrição, DietWin Plus ${ }^{\circledR}$, tendo sido possível identificar a prevalência de adequação do consumo lipídico da população. Para a classificação de adequação do consumo lipídico total diário, ácido graxo monoinsaturado e poli-insaturado foram utilizadas as recomendações da IV Diretriz Brasileira sobre Dislipidemias e Prevenção da Aterosclerose (2007), a qual recomenda que a ingestão diária das respectivas frações lipídicas sejam equivalentes a $25 \%$ até $35 \%$ do valor energético total (VET) de lipídeos totais, $\leq 20 \%$ do VET de ácido graxo monoinsaturado e $\leq 10 \%$ do VET de ácido graxo poli-insaturado. Para a classificação, quanto à adequação do consumo de colesterol, gordura saturada e a relação de consumo de ômega 6 : ômega 3 , foi utilizada a I Diretriz Sobre o Consumo de Gorduras e Saúde Cardiovascular (2013), que recomenda um consumo diário de colesterol $<300 \mathrm{mg}$, gordura saturada $<10 \%$ do VET e relação ômega6: ômega 3 de 7:1.

As análises estatísticas para comparar a população das três cidades estudadas foram feitas por testes Kruskal-Wallis Test. Para as variáveis classificatórias descritivas (idade, sexo, escolaridade, tempo de estudo, renda, se aposentados, com quem reside, tipo de residência, IMC e circunferência da cintura), foram empregadas tabelas contendo frequências absolutas (n) e relativas (\%), para média, mediana, desvio-padrão e intervalos inter-quartis. Para analisar as diferenças e comparar o consumo lipídico entre as variáveis listadas anteriormente, foi utilizado o Teste Qui-Quadrado de Pearson. 
O nível de significância foi determinado em $\mathrm{p}<0,05$.

O Comitê de Ética e Pesquisa (CEP) da Universidade Feevale, sob processo número 4.06.01.10.1729, aprovou o projeto de pesquisa o qual este estudo está relacionado e todos os participantes assinaram o Termo de Consentimento Livre e Esclarecido (TCLE).

\section{Resultados}

De um universo de 526 prontuários de idosos do banco de dados, depois de aplicados os critérios de inclusão e exclusão, a amostra apresentou um total de 482 prontuários de idosos, uma vez que os demais registros apresentaram informações imprecisas ou incompletas. Quanto às cidades, Novo Hamburgo correspondeu a $47,5 \%$ da amostra ( $n=229$ ), seguido de Estância Velha, a qual compreendia $35,9 \%$ da população $(\mathrm{n}=173)$ e Campo Bom contribuiu com $16,6 \%(n=80)$. As características sociodemográficas da amostra estão descritas na Tabela 1.
Tabela 1 - Características sociodemográficas de idosos residentes em Novo Hamburgo, Estância Velha e Campo Bom

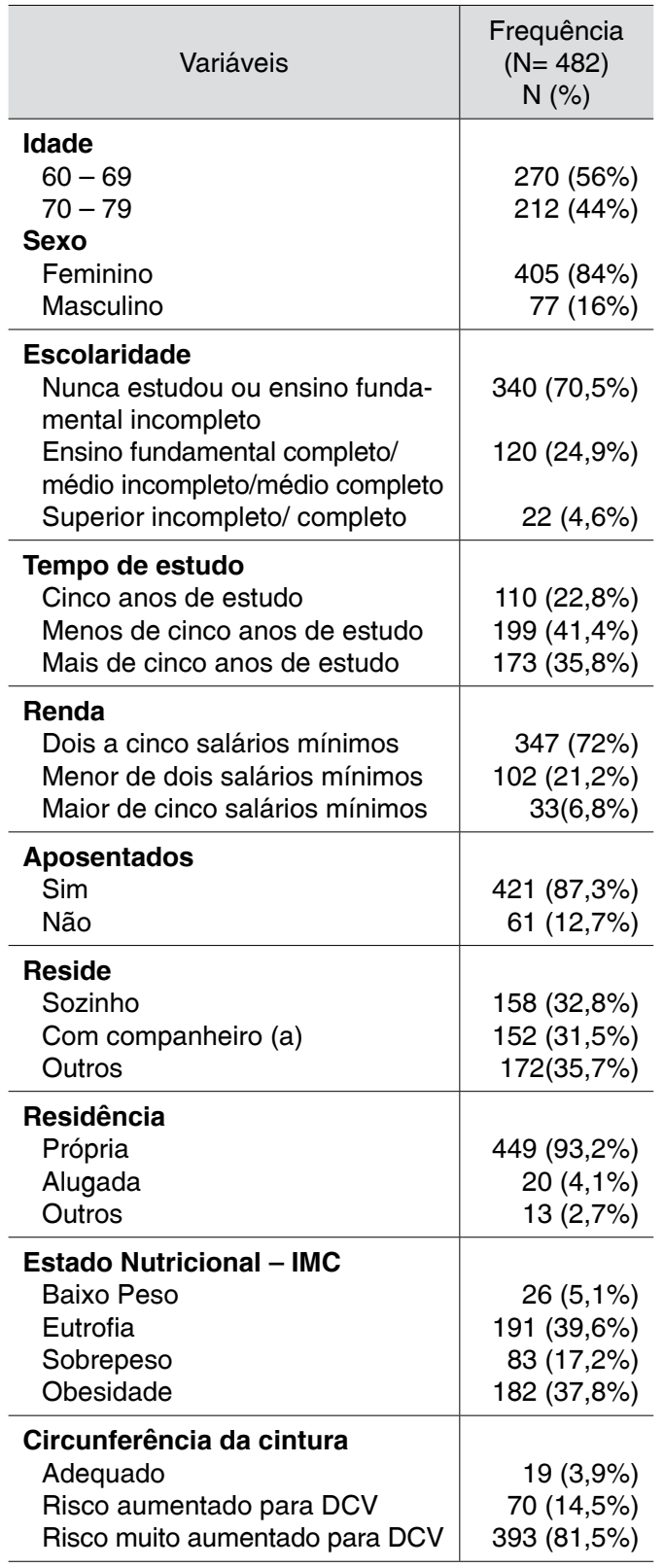

Fonte: elaboração dos autores. 
A Tabela 2 apresenta o consumo lipídico da população geral, mostrando resultado de inadequação de consumo apenas para a relação ômega 6: ômega 3 , em que recomenda-se que o valor máximo de ingestão não deva ultrapassar a relação de 7:1. Para os demais consumos de ácidos graxos, a população mostrou níveis adequados, salientando que foram usados valores de referências para indivíduos saudáveis. No entanto, observou-se pelo recordatório alimentar que os alimentos fontes de lipídeos mais consumidos entre os idosos eram, apresuntados, linguiça, nata, margarina, óleo de soja, banha de porco, entre outros.

Tabela 2 - Avaliação do consumo dietético de ácidos graxos referente à amostra estudada e percentual de adequação de consumo

\begin{tabular}{l|r|r|r|r}
\cline { 2 - 4 } & \multicolumn{1}{c|}{ Mediana (p25-p75) } & \multicolumn{1}{c|}{ Min - Máx. } & Recomendado* & \% Adequação \\
\hline VET (kcal) & $1318,37(1054-1640)$ & $469,7-6206,0$ & - & - \\
Lipídio (gramas) & $36,63(26,12-52,66)$ & $4,99-255,33$ & - & - \\
Lipídio (\%) & $25,54(20,37-31,49)$ & $4,51-93,51$ & $25-35 \%$ & - \\
Saturado (\%) & $8,88(6,52-11,67)$ & $0,66-29,94$ & $<10 \%$ & $58,5 \%$ \\
Poli-insaturado (\%) & $4,90(2,99-6,85)$ & $0,47-52,60$ & $\leq 10 \%$ & $91,9 \%$ \\
Monoinsaturado (\%) & $6,59(4,80-8,82)$ & $0,12-34,58$ & $\leq 20 \%$ & $99,2 \%$ \\
Ômega 6: ômega 3 & $9,4: 1(6,60-15,03)$ & $0,14: 1-22,4: 1$ & $7: 1$ & $27,2 \%$ \\
Colesterol (mg) & $151,76(96,66-245,23)$ & $5-2232,7$ & $<300 \mathrm{mg}$ & $82,2 \%$ \\
\hline
\end{tabular}

Fonte: elaboração dos autores.

Com base na I Diretriz sobre o consumo de Gorduras e Saúde Cardiovascular (SANTOS et al. 2013) para as recomendações de colesterol, relação ômega 6 : ômega 3 e gordura saturada. Para as recomendações de lipídeo total, gordura monoinsaturada e poli-insaturada, foi utilizada a IV Diretriz Brasileira sobre Dislipidemias e Prevenção de Aterosclerose (SPOSITO et al., 2007). A avaliação de adequação do consumo lipídico, quan- do estratificado por cidades, evidenciou que a cidade de Campo Bom apresentou melhores níveis desse quando comparada a Novo Hamburgo e Estância Velha, conforme Tabela 3. No entanto, houve diferença estatisticamente significativa apenas para a relação de consumo de ômega 6: ômega 3, mostrando-se significativamente melhor $(p=0,002)$ na cidade de Campo Bom quando comparadas as demais cidades. 
Tabela 3 - Análise de adequação do consumo de ácido graxo saturado, poli-insaturado, monoinsaturado, relação ômega 6: ômega 3 e colesterol, por cidade de residência

\begin{tabular}{|c|c|c|c|c|}
\hline & & & & \\
\hline & Novo Hamburgo & Estância Velha & Campo Bom & $\mathrm{P}^{*}$ \\
\hline \multicolumn{5}{|l|}{ Saturado } \\
\hline Mediana (P25 - 75) & $9,40(6,84-11,93)$ & $8,80(6,75-11,66)$ & $7,61(5,90-10,82)$ & 0,09 \\
\hline \% Adequação & $124(54,1 \%)$ & $104(60,1 \%)$ & $54(67,5 \%)$ & \\
\hline \multicolumn{5}{|l|}{ Poli-insaturado } \\
\hline Mediana (P25 - 75) & $5,25(3,22-7,37)$ & $4,89(3,10-6,58)$ & $3,74(2,32-5,78)$ & 0,29 \\
\hline \% Adequação & $206(90 \%)$ & $163(94,2 \%)$ & $74(92,5 \%)$ & \\
\hline \multicolumn{5}{|l|}{ Monoinsaturado } \\
\hline Mediana (P25 -75) & $6,68(4,99-9,25)$ & $7,74(5,27-8,76)$ & $5,51(3,96-8,24)$ & 0,86 \\
\hline \% Adequação & $227(99,1 \%)$ & $172(99,4 \%)$ & $79(98,8 \%)$ & \\
\hline \multicolumn{5}{|l|}{ Ômega 6: Ômega 3} \\
\hline Mediana (P25 - 75) & $10,97: 1(8,16-21,85)$ & $8,22: 1(6,21-11,15)$ & $7,85: 1(5,05-10,62)$ & 0,002 \\
\hline \% Adequação & $46(20,1 \%)$ & $54(31,2 \%)$ & $31(38,9 \%)$ & \\
\hline \multicolumn{5}{|l|}{ Colesterol } \\
\hline Mediana (P25 - P75) & $166,5(91,3-245)$ & $150,5(104,8-245,28)$ & $138,29(93,84-245,82)$ & 0,91 \\
\hline \% Adequação & $187(81,7 \%)$ & $142(82,1 \%)$ & $67(83,8 \%)$ & \\
\hline \multicolumn{5}{|l|}{ Saturado } \\
\hline Mediana (P25 - 75) & $9,40(6,84-11,93)$ & $8,80(6,75-11,66)$ & $7,61(5,90-10,82)$ & 0,09 \\
\hline \% Adequação & $54,1 \%$ & $60,1 \%$ & $67,5 \%$ & \\
\hline \multicolumn{5}{|l|}{ Poli-insaturado } \\
\hline Mediana (P25 - 75) & $5,25(3,22-7,37)$ & $4,89(3,10-6,58)$ & $3,74(2,32-5,78)$ & 0,29 \\
\hline \% Adequação & $90 \%$ & $94,2 \%$ & $92,5 \%$ & \\
\hline \multicolumn{5}{|l|}{ Monoinsaturado } \\
\hline Mediana (P25 -75) & $6,68(4,99-9,25)$ & $7,74(5,27-8,76)$ & $5,51(3,96-8,24)$ & 0,86 \\
\hline \% Adequação & $99,1 \%$ & $99,4 \%$ & $98,8 \%$ & \\
\hline \multicolumn{5}{|l|}{ Ômega 6: Ômega 3} \\
\hline Mediana (P25 - 75) & $10,97: 1(8,16-21,85)$ & $8,22: 1(6,21-11,15)$ & $7,85: 1(5,05-10,62)$ & 0,002 \\
\hline \% Adequação & $20,1 \%$ & $31,2 \%$ & $38,9 \%$ & \\
\hline \multicolumn{5}{|l|}{ Colesterol } \\
\hline Mediana (P25 - P75) & $166,5(91,3-245)$ & $150,5(104,8-245,28)$ & $138,29(93,84-245,82)$ & 0,91 \\
\hline \% Adequação & $81,7 \%$ & $82,1 \%$ & $83,8 \%$ & \\
\hline
\end{tabular}

Fonte: elaboração dos autores.

Nota:* Kruskal-Wallis Test.

Por meio das análises, a relação do consumo lipídico entre os sexos, idade, IMC, circunferência da cintura, renda e escolaridade não apresentaram di- ferença estatisticamente significativa, quando comparados com a ingestão de ácidos graxos, não influenciando assim nos níveis de consumo lipídico. 


\section{Discussão}

No presente estudo, foram avaliados 482 idosos, com predomínio do sexo feminino (84\%). Essa prevalência do sexo feminino sugere que as mulheres são mais participativas nos grupos de convivência e atividades que envolvam a promoção da saúde. Esse fato corrobora a maioria dos estudos existentes que envolvam idosos, mostrando que a maioria apresenta prevalência do sexo feminino. Neri e Vieira (2013) ainda sugerem que as mulheres cultivam mais as relações sociais quando comparadas aos homens, que restringem suas atividades sociais a membros próximos, participando menos de grupos de convivência.

Corroborando o achado deste estudo, Kucera, Siviero e Bonatto (2012) que avaliaram o consumo lipídico e estado nutricional de idosos de ambos os sexos e participantes de um projeto realizado na Universidade de Caxias do Sul - RS, observaram prevalência de idosos com obesidade $(56,7 \%)$ e circunferência da cintura com risco muito aumentado para doenças cardiovasculares $(71,1 \%)$. Assim como Venturini et al. (2013), que avaliaram a prevalência de obesidade associada ao perfil lipídico de idosos residentes em Porto Alegre - RS e observou que $72 \%$ da sua amostra estava acima do peso, $35 \%$ das mulheres seguido de $21 \%$ dos homens estavam obesos. Diferentes de Brito-Gomes et al. (2015) que avaliaram modificações no IMC de mulheres idosas após um programa de reabilitação física e encontraram uma maioria eutrófica tanto antes quanto após a intervenção, passando de $70 \%$ para $74,4 \%$ da amostra.

É importante ressaltar que o estado nutricional dos idosos é um fator de risco para o desenvolvimento de doenças crônicas não transmissíveis que está diretamente ligado ao consumo alimentar, à obtenção e ao modo de preparo desses alimentos. (ANGST et al., 2015). No entanto, o risco de doenças cardiovasculares não está relacionado somente à quantidade total de gordura presente em um organismo, mas principalmente pelo modo como essa gordura se distribui pelo corpo. Nesse contexto, a obesidade padrão andróide, com maior acúmulo de gordura na região abdominal, é considerada a que oferece maior risco para a saúde, pois ela indica aumento das chances do indivíduo desenvolver doenças cardiovasculares, entre elas, infarto agudo do miocárdio e acidente vascular cerebral (KLEIN; OLIVEIRA, 2012). O presente estudo mostra grande prevalência de idosos com risco muito aumentado para DCVs de acordo com a circunferência da cintura. Apesar das alterações na composição corporal ser um fator intrínseco do processo de envelhecimento, devemos considerar que esse é individual e ocorre distintamente entre homens e mulheres, pois a redistribuição e internalização da gordura abdominal, fato que ocorre principalmente entre as idosas (PREVIATO et al., 2014).

Um dos principais achados deste estudo foi a avaliação do consumo lipídico dos idosos, que demonstrou níveis de consumo adequado em relação ao percentual total de lipídeos ingerido diaria- 
mente, além de ingestão adequada para ácido graxo saturado, poli-insaturado, monoinsaturado e colesterol. No consumo lipídico, a relação ômega 6: ômega 3 apresentou inadequação, estando acima das recomendações de consumo. Esse fato deve-se ao alto consumo de alimentos ricos em ômega 6 , como óleo de soja, e em contrapartida, escassez de alimentos fonte de ômega 3 , por exemplo, sardinha, salmão, óleo de linhaça, entre outros. Essa inadequação de consumo confere grande risco à saúde do indivíduo, aumentando a probabilidade de desenvolver doenças cardiovasculares e cardiometabólicas. De acordo com Santos et al. (2013), o ômega 3 possui um efeito cardioprotetor, exercendo inúmeros efeitos sobre aspectos fisiológica e metabólicos que estão diretamente relacionados com o desenvolvimento de doenças cardiovasculares, tais como, melhora da função autonômica cardíaca, antiarrítmico, diminuição da agregação plaquetária e da pressão arterial, melhora dos níveis plasmáticos de triglicérides, melhora da função endotelial, diminui o processo de inflamação, agindo como um estabilizante da placa de ateroma. Em contrapartida, o ômega 6 reduz níveis plasmáticos de colesterol total e LDL-C, porém, reduz drasticamente os níveis plasmáticos de HDL-C, o qual possui um efeito cardioprotetor. Estudos indicam que o ômega 6 poderia elevar a geração de medidores inflamatórios, corroborando com diversos processos patológicos, incluindo eventos cardiovasculares. Entretanto, atualmente, existem evidências substanciais de que um aumento no consumo de ômega 3 pode conferir um efeito cardioprotetor, porém, a isolada redução do consumo de ômega 6 não confere o mesmo efeito, aumentando ainda mais as chances de eventos cardíacos. Portanto, o recomendado é que se tenha um consumo adequado dessa relação, pois somente assim é possível obter resultados positivos quando relacionado ao risco de desenvolvimento de doenças cardiovasculares. $O$ fato de se tratar de uma população idosa, com hábitos alimentares cultivados durante a vida inteira, que moram em uma região do país onde, culturalmente, não se consomem alimentos fonte de ômega 3 pode ter colaborado para a inadequação do consumo dessa relação.

Em relação ao consumo das demais frações lipídicas observadas, todas encontravam-se dentro dos níveis de adequação. Indo ao encontro dos resultados obtidos nos referentes estudos, em que, Kucera, Siviera e Bonatto (2012), utilizando o R24h para avaliar o consumo alimentar de lipídeos, mostraram que a grande maioria dos idosos também consumiam níveis que estavam dentro do recomendado para ácido graxo saturado, poli-insaturado, monoinsaturado e colesterol, porém a ingestão total de lipídeos em percentual diário estava abaixo das recomendações. Benetti, Ceni (2012) observaram em uma população de idosos residentes da cidade de Erechim - RS, e observaram por meio do R24h que as mulheres possuíam um consumo diário de ácidos graxos saturados em quantidades maiores quando comparadas ao consumido pelos homens. O con- 
sumo de ácidos graxos poli-insaturados e monoinsaturados estavam dentro das recomendações diárias e o consumo de colesterol estava abaixo do recomendado. Já Abreu et al. (2013) realizaram um estudo com idosos residentes de Alto Jequitibá - MG e observaram, por meio da aplicação do questionário de frequência alimentar, que aquela amostra possuía baixo consumo de colesterol e adequado consumo de ácidos graxos saturados. No entanto os autores ressaltam que o método utilizado para avaliação do consumo alimentar e as tabelas utilizadas para os cálculos de colesterol podem ter superestimado o consumo desses nutrientes, interferindo, assim, no resultado final, agindo como limitante do estudo. Esses resultados podem ter sido influenciados pelo instrumento usado para mensurar o consumo alimentar diário, os quais foram utilizados R24h e questionário de frequência alimentar, porém, o presente estudo teve um número amostral muito maior que os estudos supracitados, o que pode reforçar a veracidade dos resultados finais.

Dentre as cidades avaliadas neste estudo, os idosos residentes em Campo Bom apresentaram melhor consumo lipídico em todas as frações analisadas. Pesquisas realizadas mostraram que as três cidades foram colonizadas por imigrantes germânicos, logo, não se pode atribuir esse fato a diferença no consumo lipídico. No entanto, a cidade de Novo Hamburgo apresenta uma mescla maior de outras culturas, além de ser uma cidade mais "urbanizada", o que pode influenciar uma alimentação com maior consumo de fast foods e produtos industrializados. De acordo com o recordatório alimentar de Estância Velha pôde-se perceber que estes idosos possuem hábito de consumir produtos como banha de porco e nata, no entanto, produtos caseiros fazem parte da sua rotina alimentar, o que pode ter corroborado com os resultados obtidos nesta pesquisa, os quais mostraram que a maioria da população estudada apresentou inadequação de consumo apenas para a relação ômega 6:ômega 3. A cidade de Campo Bom, por sua vez, destaca-se como a maior produtora de mudas de hortaliças do Rio Grande do Sul, podendo estar relacionada a um consumo maior desses produtos pela população, avaliados por meio do R24h, e consequentemente, menor consumo de alimentos com alto teor lipídico.

$\mathrm{O}$ presente estudo apresentou alguns fatores limitantes como, o instrumento utilizado para mensuração do consumo alimentar, recordatório de 24 horas, que pode ter subestimado o consumo alimentar dos idosos e não caracteriza padrão alimentar. Segundo Pedreza e Menezes (2015), após avaliarem estudos que desenvolveram e validaram instrumentos de avaliação do consumo alimentar no Brasil, como questionário de frequência alimentar e R24h, apontaram que R24h foi o método de avaliação dietética mais usado como padrão de referência para o estabelecimento de erros sistemáticos. Outro aspecto limitante pode ter sido o fato de se tratar de um estudo transversal, observando esses idosos em um único momento, podendo aumentar as chances de ocorrer um viés de causalida- 
de reversa. A dificuldade de expressar e interpretar o tamanho da porção caseira do alimento consumido, não foi considerado neste estudo, uma vez que os idosos obtiveram auxílio de instrumentos ilustrativos para a definição das porções.

No Brasil ainda existem poucos estudos que expressam o consumo alimentar e perfil lipídico da população idosa, o que ressalta a importância de novos estudos que avaliem sua ingestão alimentar por meio de instrumentos específicos para essa população, para que se possa confirmar os resultados obtidos e, assim, criar estratégias para promoção da saúde e prevenção de doenças por meio de um acompanhamento nutricional e um controle dietético (PREVIATTO et al., 2015).

\section{Conclusão}

Por meio deste estudo pode-se concluir que apesar de os idosos residentes na cidade de Campo Bom apresentar níveis mais baixos de consumo lipídico quando comparadas às demais cidades, todas estavam dentro da faixa de normalidade, exceto para o consumo ômega 6: ômega 3. Ressalta-se que a cidade de Novo Hamburgo apresentou os maiores níveis de consumo lipídico, com consumo próximo do máximo recomendado, além de obter a maior inadequação do consumo de ômega 6: ômega 3.

Salienta-se, então, que o papel do nutricionista é de suma importância para que haja um consumo equilibrado de todos os nutrientes, estimulando a ingestão de alimentos cardioprotetores, principalmente na população idosa.
Conclui-se que são necessárias mais pesquisas que avaliem a ingestão lipídica em idosos, a fim de comparar e discutir os resultados obtidos neste estudo.

\section{Analysis of the lipid consumption of elderly residents of the Sinos Valley Region, RS}

\section{Abstract}

Cross - sectional and retrospective study, aiming to know the dietary intake of fatty acids in the elderly, correlating with sex, age, city of residence and nutritional status. A total of 526 medical records of elderly people participating in a study entitled "Aging and Quality of Life: A Study in Selected Municipalities of Vale dos Sinos, RS" were used, which, after being evaluated by the inclusion and exclusion criteria, resulted in 482 medical records used in this study. The research was carried out between 2011 and 2014 with elderly of both sexes, aged between 60 and 79 years, residents of Campo Bom, Estância Velha or Novo Hamburgo - RS. Sociodemographic, anthropometric and food consumption data (R24h) were evaluated. The sample was predominantly female $(84 \%)$, retired elderly $(87.3 \%)$, income from two to five minimum wages $(72 \%)$, schooling less than complete elementary education $(70.5 \%)$, less than five years of schooling $(41.4 \%)$, were accompanied (35.7\%), home $(93.2 \%)$, predominance of overweight $(55 \%)$ and very increased risk for cardiovascular disease $(81,5 \%)$. We can consider after the analysis of the results, that the consumption of fatty acids of the elderly of the studied region, was adequate within the range of normality, except for the ratio of consumption of omega 6: 3 . The city of Campo Bom showed better levels 
of fatty acid consumption in all lipid fractions, with significant results for the omega 6: 3 consumption ratio $(p=0.002)$. The other variables did not present significant results in relation to lipid consumption.

Keywords: Elderly. Fatty acids. Cardiovascular diseases.

\section{Agradecimentos}

Agradeço a Instituição Universidade Feevale, que disponibilizou espaço para coleta dos dados, aos professores envolvidos e à todas as demais pessoas que contribuíram para o desenvolvimento desta pesquisa.

\section{Referências}

ABESO - Associação Brasileira para o Estudo da Obesidade e da Síndrome Metabólica Diretrizes brasileiras de obesidade 2009/2010 - Associação Brasileira para o Estudo da Obesidade e da Síndrome Metabólica. - 3.ed. - Itapevi (SP): AC Farmacêutica, 2009.

ABREU, I. C. M. E. et al. Baixa renda de idosos está associada à qualidade da dieta. Alimimentos e Nutrição: Brazilian Journal of Food and Nutrition, Araraquara (SP), v. 24, n. 3, p. 349-357, jul.-set. 2013.

ANGST, C. A. et al. Estado nutricional em idosos grupos de convivência. Revista Brasileira de Ciências do Envelhecimento Humano, Passo Fundo (RS), v. 12, n. 1, p. 41-55, jan.-abr. 2015

BENETTI, F.; CENI, G. C. Hábitos alimentares de idosos portadores de diabetes: relação entre ingesta alimentar e recomendações nutricionais. Revista Brasileira de Ciências do Envelhecimento Humano, Passo Fundo (RS), v. 9, n. 3, p. 383-394, set.-dez. 2012.
BEZERRA, F. C.; ALMEIDA, M. I.; THERRIEN-NÓBREGA, S. M. Estudos sobre Envelhecimento no Brasil: Revisão Bibliográfica. Revista Brasileira de Geriatria e Gerontologia, Rio De Janeiro (RJ), v. 15, n. 1, p. 155-167, ago.-dez. 2012.

BRITO-GOMES, J.L. et al. Modificações no índice de massa corporal em mulheres idosas. Revista de Terapia Ocupacional da Universidade de São Paulo, São Paulo (SP), v. 26 , n. 1, p. 146-152, jan.-abr. 2015.

HERDY, A. H. et al. Sociedade Brasileira de Cardiologia. Diretriz Sul-Americana de Prevenção e Reabilitação Cardiovascular. Arquivos Brasileiros de Cardiologia, Rio de Janeiro (RJ), v. 103, n. 2, Supl. 1, p. 1-31, jan. 2015.

IBGE. Instituto Brasileiro de Geografia e Estatística. Síntese de Indicadores Sociais: uma Análise das Condições de Vida da População Brasileira 2013. Rio de Janeiro (RJ), 2013.

IBGE. Instituto Brasileiro de Geografia e Estatística. Pesquisa Nacional de Saúde 2013: Percepção do estado de saúde, estilos de vida e doenças crônicas. Rio de Janeiro (RJ), 2013.

KLEIN K.B., OLIVEIRA T.B. Avaliação dos fatores de risco para doenças cardiovasculares em idosos participantes do projeto viva a vida no município de Santo Ângelo, RS. $R e$ vista Brasileira de Farmácia, Rio de Janeiro (RJ), v. 93, n. 2, p. 215-220, fev./mar. 2012.

KUCERA, M. O. R.; SIVIERO, J.; BONATTO, S. Consumo de Lipídeos e Estado Nutricional de Idosos Participantes do Projeto NUTENV da Universidade de Caxias do Sul. Revista Brasileira de Ciências do Envelhecimento Humano, Passo Fundo (RS), v. 9, n. 3, p. 426-438, set.-dez. 2012.

MUNICÍPIO DE CAMPO BOM. Dados Gerais do Município. Campo Bom (RS), 2012. Disponível em: <http://novo.campobom. rs.gov.br/informacoes/dados-gerais-do-municipio>. Acesso em: 15 maio 2016. 
MUNICÍPIO DE ESTÂNCIA VELHA. História: primeiros moradores. Estância Velha (RS), 2015. Disponível em: <http://www. estanciavelha.rs.gov.br/historia>. Acesso em: 15 maio 2016.

MUNICÍPIO DE NOVO HAMBURGO. História: Novo Hamburgo, passado e futuro. Novo Hamburgo (RS), 2014. Disponível em: < http://www.novohamburgo.rs.gov.br/ modules/catasg/novohamburgo.php?conteudo=70>. Acesso em: 15 maio 2016.

NERI, A. L; VIEIRA, L. A. M. Envolvimento social e suporte social percebido na velhice. Revista Brasileira de Geriatria e Gerontologia, Rio de Janeiro (RJ), v. 16, n. 3, p. 419432, set. 2013.

NEVES, R. T. et al. Envelhecimento e doenças cardiovasculares: depressão e qualidade de vida em idosos atendidos em domicílio. Psicologia Hospitalar, São Paulo (SP), v. 11, n. 2 , p. $45-57$, jul. 2013

OPAS - Organização Pan-Americana da Saúde. O projeto $S A B E$ no município de São Paulo: uma abordagem inicial/ Maria Lúcia Lebrão, Yeda A. de Oliveira Duarte. Brasília (DF), 2003.

PEDRAZA, D.F; MENEZES, T. N. Questionários de Frequência de Consumo Alimentar desenvolvidos e validados para população do Brasil: revisão da literatura. Ciência \& Saúde Coletiva, Rio de Janeiro (RJ), v. 20, n. 9, p. 2697-2720, set. 2015.

PREVIATTO, H. D. R. A. et al. Perfil clínico-nutricional e consumo alimentar de idosos do Programa Terceira Idade, Ouro Preto-MG. Demetra, Rio de Janeiro (RJ), v. 10, n. 2, p. 375-387, out. 2015.

PREVIATO, H. D. R. A. et al. Associação entre índice de massas corporal e circunferência da cintura em idosas, Ouro Preto, Minas Gerais, Brasil. Nutrícion Clínica y Dietética Hospitalaria, Madrid (Espanha), v. 34, n. 1, p. 25-30, maio 2014.
SANTOS, R. D. et al. I Diretriz sobre o consumo de gorduras e saúde cardiovascular. Arquivos Brasileiros de Cardiologia, São Paulo (SP), v. 100, n. 1, supl. 3, p. 1-40, jan. 2013.

SPOSITO et al., A. C. IV Diretriz Brasileira sobre Dislipidemias e Prevenção da Aterosclerose: Departamento de Aterosclerose da Sociedade Brasileira de Cardiologia. Arquivos Brasileiros de Cardiologia, São Paulo (SP), v. 88, supl. 1, p. 2-19, abr. 2007.

VENTURINI, C. D. et al. Prevalência de obesidade associada à ingestão calórica, glicemia e perfil lipídico em uma amostra populacional de idosos do Sul do Brasil. Revista Brasileira de Geriatria e Gerontologia, Rio de Janeiro (RJ), v. 16, n. 3, p. 591-601, set. 2013. 